\title{
Leveraging the Economic Potential of FCC's Technologies and Processes
}

\author{
Linn Kretzschmar
}

\begin{abstract}
Contents

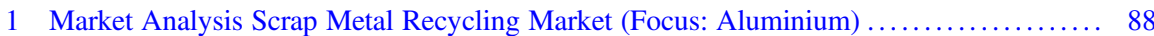

2 Market Analysis: NMR Technology in the Fruit Sorting Industry ................... 89

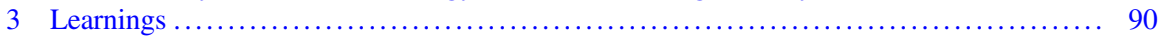

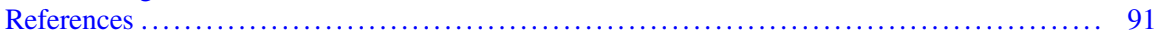

An international consortium of more than 150 organizations worldwide is studying the feasibility of various future particle colliders to expand our understanding of the inner workings of the Universe. At the core of the Future Circular Collider (FCC) study is the design of a $100 \mathrm{~km}$ long circular particle collider infrastructure that could extend CERN's current accelerator complex with an integral research program that spans 70 years. The first step would be an intensity-frontier electron-positron collider allowing to study with precision the Higgs couplings with many of the Standard Model particles and search with high-precision for new physics while the ultimate goal is to build a proton collider with a c.m.s collision energy seven times larger than the Large Hadron Collider. Hosted in the same tunnel and profiting from the new infrastructure, FCC-hh would allow to explore a new energy regime where new physics may be at play.

One of the technologies lying at the heart of energy-frontier colliders is superconductivity. The dipole magnets for steering the charged particles in their circular trajectories call for superconducting magnet technology that remains yet to be developed while this technology should be available at large scales as required by the size of a new collider-based RI. Since it takes decades for such a technology to reach industrial maturity, research and development efforts are well underway.

In this context and starting in 2017, the Institute for Entrepreneurship and Innovation at the Vienna University of Economics and Business conducted three research projects to analyze the potential of new application fields outside of the particle collider domain for superconducting magnets and important steps of their

L. Kretzschmar $(\square)$

MSCA EASITrain Fellow, University of Economics, Vienna, Austria

e-mail: linn.kretzschmar@wu.ac.at

(C) The Author(s) 2021

H. P. Beck, P. Charitos (eds.), The Economics of Big Science, Science Policy

Reports, https://doi.org/10.1007/978-3-030-52391-6_12 
manufacturing value chain [1]. The overarching question behind these research projects is how, apart from learning more about the universe, society can benefit from a new research infrastructure during the design, construction and operation phase. In order to exploit the value of superconducting technologies utilized to build an electron-positron collider for other industrial purposes, the knowledge acquired from manufacturing these technologies needs to be spread and appropriated. To achieve this goal, the Institute of Entrepreneurship and Innovation at Vienna University of Economics (WUW), under the lead of CERN, identified innovative application fields outside of the particle collider domain for superconducting magnets the processes involved in manufacturing those technologies and assessed these new application fields and conducted elaborate market analyses for the most promising ones. For a detailed description of the projects, see [2] and [3]. The following paragraphs outline the methodology and main findings of these projects.

To identify new market opportunities for superconducting magnets and its manufacturing steps, the manufacturing value chain needed to be analyzed with regard to their importance. Based on factors such as uniqueness and cost intensity, the superconducting Rutherford cable, the furnace for the thermal treatment as well as the CTD-101 k epoxy resin for vacuum impregnation of the superconducting magnets were identified as the three most valuable parts of the manufacturing process. Subsequently, a method called Technology Competence Leveraging (TCL) was utilized to identify new application fields for each of the selected high technologies and processes. TCL is based on open innovation principles such as crowdsourcing and combines creative and analytical methods to systematically trigger the discovery of new fields of applications in four sequential steps [4]. Following this method, the first step was to identify the key use benefits of these technologies by interviewing experts and actual users. After the identification of the respective use benefits, the goal was to identify as many new application fields as possible by divergent thinking with creativity techniques such as brainstorming and searching for industries that seek to solve similar problems like the ones solved by the technologies. The ideas were clustered and evaluated based on interviews with potential users to assure their applicability. Once a list of probable application fields was established, they were evaluated according to their benefit relevance and strategic fit. These concepts rate the ideas concerning their marketability. While benefit relevance assesses the relevance of the problem to be solved, strategic fit measures the fit of a given idea to the producing company and its resources, capabilities and culture.

For the final step in TCL, the market potential was analyzed by identifying how to derive value from a particular idea, for whom, how to generate income and which key partners are needed to implement the idea. Based on the analysis, the market potential of a new application field could be estimated. An overview of the 21 most promising application fields can be found in Fig. 1 below.

TCL proved to be a very useful tool to identify new market opportunities for technologies involved in manufacturing superconducting magnets. In total, 65 new application fields could be identified using this method. For two of the potential application fields, scrap metal recycling and fruit sorting, a detailed market analysis was conducted in a follow-up project. An overview about the results can be found in the following paragraphs. 


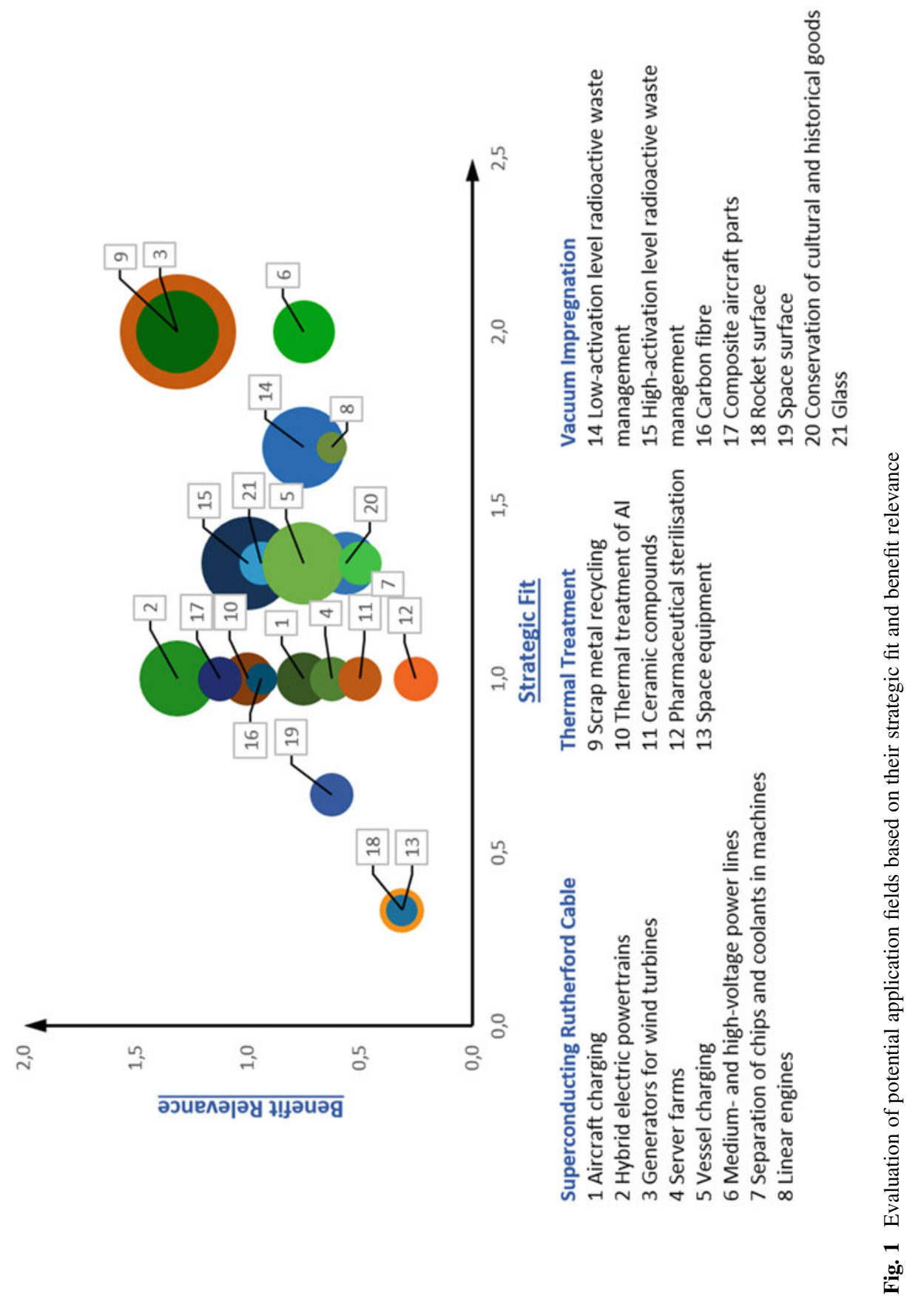




\section{Market Analysis Scrap Metal Recycling Market (Focus: Aluminium)}

One potential application field of the furnace used to treat the Rutherford cables is the use in the smelting industry. Compared to the standard furnaces in the industry, it has the advantage that it can reach high temperatures up to $900{ }^{\circ} \mathrm{C}$ precisely within an accuracy range of $\pm 3{ }^{\circ} \mathrm{C}$. In comparison, furnaces used in the steel industry have a range of $\pm 10{ }^{\circ} \mathrm{C}$. Furthermore, the heating system generates a homogenous diffusion of heat with internal fans and permits fast temperature changes at a rate of $50 \mathrm{~K}$ per hour. By leveraging the protective properties of argon, a noble gas, the furnace additionally minimizes the shrinking of metals due to corrosion (oxidation). This way, the process becomes more efficient and less material is lost, which is important for valuable and expensive materials. These properties are particularly relevant for the metal recycling industry. Utilizing the above-mentioned furnace can reduce the number of steps by combining the splitting up and melting of several metals. Specifically, the technology allows the separation of metals with different melting points. According to interviews conducted with industry experts, the furnace is particularly attractive for the aluminium recycling industry. Currently, 60 Million tons of aluminium are used annually and the consumption is predicted to increase by $18 \%$ until 2023. The global demand for this valuable metal is primarily driven by industries such as construction, aviation and automotive, where it has replaced steel due to its high strength to weight ratio. The metal is highly recyclable, with around $75 \%$ of all aluminium manufactured, dating as far back as 125 years, still being in use today. The metal is infinitely recyclable without degradation and its recycling process is considered to be closed-looped. Furthermore, the re-melting process of aluminum is much less costly and energy-intensive, using only $5 \%$ of the energy needed for the production of new aluminum.

The current recycling process exhibits some challenges such as possible contamination. Impurities in the metal have an impact on its properties and can weaken the metal, rendering it unusable for specific industrial purposes. However, by using the particular furnace for the process, the aluminum could be separated from contaminating metals such as lead and tin, which are primarily responsible for its contamination. Furthermore, during the melting process, a waste by-product called dross develops due to the oxidation of the metal. With argon as a protective gas, the furnace could help to reduce the development of dross, consequently diminishing the loss of aluminum during the recycling process.

The aluminum recycling industry is steadily growing: While primary aluminum production decreased by $8.5 \%$ within 5 years, the amount of recycled aluminum increased by $21.6 \%$ within the same timeframe until 2015. In Europe alone, this secondary aluminum production is operated by more than 300 aluminum recycling plants. 


\section{Market Analysis: NMR Technology in the Fruit Sorting Industry}

Nuclear magnetic resonance spectroscopy can be utilized to grade and sort fruits and vegetables by their quality. Standard sorting machines in the food industry work with high-frequency cameras that take pictures of vegetables and fruits from different angles. This approach however limits the quality evaluation to external characteristics such as size, color, curvature and visible damages, disregarding potential internal physiological defects. According to industry experts from companies such as VOG, Microtec and Greefa, this is an important concern in the food quality industry and thus, some companies currently employ infrared and UV technology to detect internal defects. However, these technologies are not able to conduct a structural analysis of organic material, determining for example the maturity of the produce, which is an important predictor for shelf life expectancy and storage conditions. In addition to limited conclusiveness about total product quality, many technologies are invasive (thus destroying the fruit or vegetable, time-consuming or too superficial to analyze the fruit core. Nuclear magnetic resonance (NMR) spectroscopy can overcome these challenges by analyzing the chemical composition of the products and detecting parasites, impurities or diseases based on compositional and structural properties.

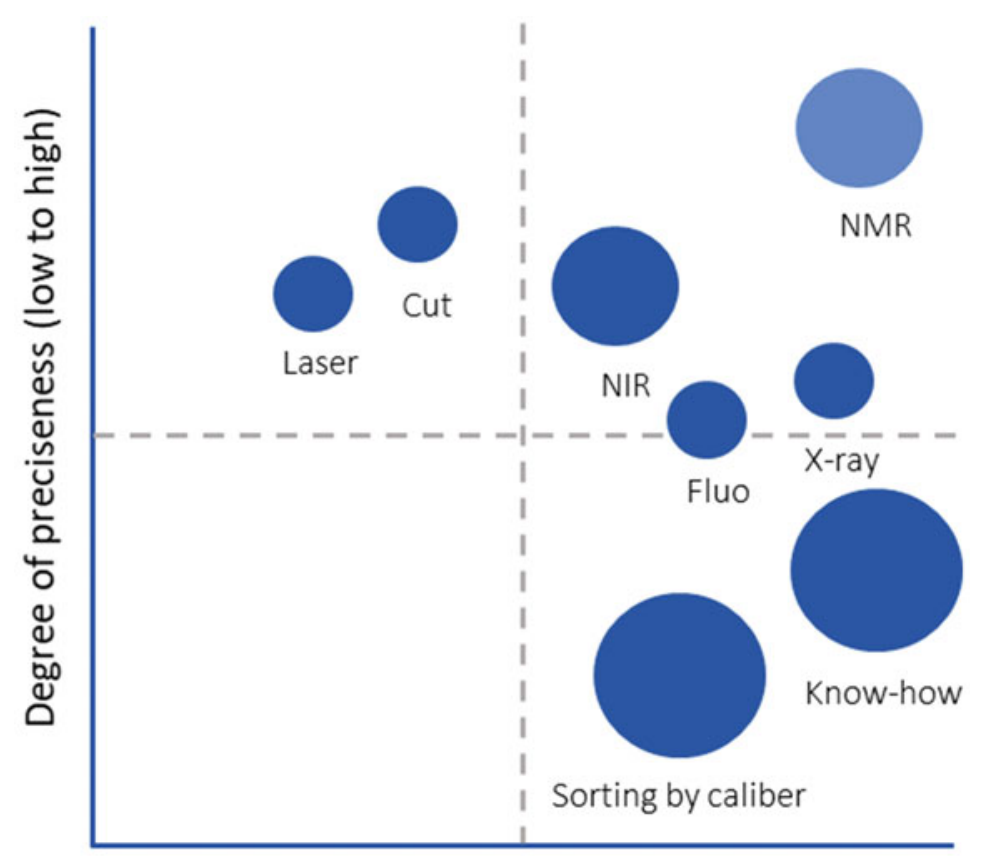

Degree of invasiveness (high to low) 
In recent years, the food sorting market has exhibited steady growth, with the average growth rate of $7.24 \%$ per year. Factors contributing to the development are higher consumer expectations as well as the demand for higher quality standards within the industry. With its non-invasive properties, NMR-based technology can ensure the quality of organic produce throughout the entire food supply chain and would enable producers to diversify their prices concerning the quality of the product. In 2008, FFV waste of US retail stores due to low quality was estimated at around USD43 Billion (retail price). Since food waste due to spoilage and contamination incurs the most economic costs at the end of the supply chain, an employment of NMR early in the value chain would economically be the most effective option.

\section{Learnings}

The research projects outlined above revealed promising application fields outside of the typical particle collider domain, which means that benefits derived from the superconducting technology research go beyond building research infrastructures to answer fundamental research questions. While not apparent at first glance, the research about new high technologies comes with profound economic impact on other industries. There are three key takeaways from conducting these projects: First, when evaluating the application potential of high technologies, it is important to consider not only the final product, but also knowledge, processes and technologies involved in manufacturing the product when assessing market potential. Many processes involved in the manufacturing process have great use benefits and thus, high application potential in other industries. Not leveraged, the industrial value of high technologies is systematically underestimated. Second, the identification of new application fields is an iterative process, including many dead ends. It is crucial for the success of such a project to anticipate unexpected turns in finding suitableand especially valuable - application fields. Finally, in order to produce accurate results, it is essential to interdisciplinary collaboration between scientists and industrial players. Without combining the knowledge from technology experts with market insights from companies and business experts, a diffusion of technological advancements from science to industry is unlikely to be successful.

Acknowledgments The research discussed in this paper is supported by the ASITrain-European Advanced Superconductivity Innovation and Training. The Marie Sklodowska-Curie Action (MSCA) Innovative Training Networks (ITN) receives funding from the European Union's H2020 Framework Programme under grant agreement No. 764879. 


\section{References}

1. Keinz, P. \& Kretzschmar, L. (2019). Impact potentials of EASITrain research on society \& industry (Version V1.0). Zenodo. https://zenodo.org/record/3458923\#.Xa8VentCRaQ

2. Brzobohaty, L., Habernig, S., Moravec, P., Pably, M., Schürz, T., Kretzschmar, L. \& Quach S. (2019). Analysis of potential markets for using technologies in the superconducting magnet value chain (Version 1.0). Zenodo. https://doi.org/10.5281/zenodo.3362855

3. Kretzschmar, L., Mehner, B., Hausberger, M., Ledermüller, F., Mayrhofer, F., Schreiber, D. and Gutleber, J. (2019). Manufacturing process of superconducting magnets: Analysis of manufacturing chain technologies for market-oriented industries (Version 1.0). Zenodo. https:// doi.org/10.5281/zenodo. 2579834

4. Keinz, P., \& Prügl, R. (2010). A user community-based approach to leveraging technological competences: An exploratory case study of a technology start-up from MIT. Creativity and Innovation Management, 19(3), 269-289.

\section{Full Presentation}

https://indico.cern.ch/event/727555/contributions/3461288/attachments/1867856/3073626/Linn_ Kretzschmar_-Leveraging_the_economic_potential_of_FCCs_technologies_and_processes. pdf

Open Access This chapter is licensed under the terms of the Creative Commons Attribution 4.0 International License (http://creativecommons.org/licenses/by/4.0/), which permits use, sharing, adaptation, distribution and reproduction in any medium or format, as long as you give appropriate credit to the original author(s) and the source, provide a link to the Creative Commons licence and indicate if changes were made.

The images or other third party material in this chapter are included in the chapter's Creative Commons licence, unless indicated otherwise in a credit line to the material. If material is not included in the chapter's Creative Commons licence and your intended use is not permitted by statutory regulation or exceeds the permitted use, you will need to obtain permission directly from the copyright holder.

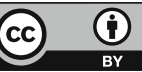

